\title{
Density matrix variational theory: Application to the potential energy surfaces and strongly correlated systems
}

\author{
$\operatorname{AUTHOR}(S)$ :
}

Nakata, M; Ehara, M; Nakatsuji, H

\section{CITATION:}

Nakata, M ...[et al]. Density matrix variational theory: Application to the potential energy surfaces and strongly correlated systems. JOURNAL OF CHEMICAL PHYSICS 2002, 116(13): 5432-5439

\section{ISSUE DATE:}

2002-04-01

URL:

http://hdl.handle.net/2433/50099

\section{RIGHT:}

Copyright 2002 American Institute of Physics. This article may be downloaded for personal use only. Any other use requires prior permission of the author and the American Institute of Physics. 


\title{
Density matrix variational theory: Application to the potential energy surfaces and strongly correlated systems
}

\author{
Maho Nakata, Masahiro Ehara, and Hiroshi Nakatsuji ${ }^{\text {a) }}$ \\ Department of Synthetic Chemistry and Biological Chemistry, Faculty of Engineering, Kyoto University, \\ Kyoto 606-8501, Japan
}

(Received 23 August 2001; accepted 7 January 2002)

\begin{abstract}
The density matrix variational theory (DMVT) algorithm developed previously [J. Chem. Phys. 114, 8282 (2001)] was utilized for calculations of the potential energy surfaces of molecules, $\mathrm{H}_{4}, \mathrm{H}_{2} \mathrm{O}$, $\mathrm{NH}_{3}, \mathrm{BH}_{3}, \mathrm{CO}, \mathrm{N}_{2}, \mathrm{C}_{2}$, and $\mathrm{Be}_{2}$. The $\operatorname{DMVT}(P Q G)$, using the $P, Q$, and $G$ conditions as subsidiary condition, reproduced the full-CI curves very accurately even up to the dissociation limit. The method described well the quasidegenerate states and the strongly correlated systems. On the other hand, the DMVT $(P Q)$ was not satisfactory especially in the dissociation limit and its potential curves were always repulsive. The size consistency of the method was discussed and the $G$ condition was found to be essential for the correct behavior of the potential curve. Further, we also examined the Weinhold-Wilson inequalities for the resultant 2-RDM of $\operatorname{DMVT}(P Q G)$ calculations. Two linear inequalities were violated when the results were less accurate, suggesting that this inequality may provide a useful $N$-representability condition for the DMVT. (C) 2002 American Institute of Physics. [DOI: 10.1063/1.1453961]
\end{abstract}

\section{INTRODUCTION}

The second-order reduced density matrix (2-RDM) completely describes the $N$-body fermion system since any observable properties of the system can be calculated from the 2-RDM. ${ }^{1,2}$ This fact has motivated us to use 2 -RDM as a basic variable of quantum mechanics instead of the wave function $\Psi$. If we can determine 2-RDM without using $\Psi$, we have a closed form of quantum mechanics where the basic variable is 2-RDM. We refer to such formalism of quantum mechanics as density matrix theory(DMT). There are two categories in the DMT with respect to the determination of the RDM. One is based on the density equation, ${ }^{3}$ which is equivalent to the Schrödinger equation in the necessary and sufficient sense. This approach is called density equation theory (DET). Recently, DET is extensively studied and developed. ${ }^{4-6}$ They have been summarized in a recent review paper. ${ }^{7}$ The other is based on the Ritz variational principle expressed in terms of 2-RDM. This latter approach is called density matrix variational theory (DMVT). The key in this approach is how well we can restrict our variable 2-RDM to be $N$-representable. ${ }^{8}$

Garrod and Percus ${ }^{9}$ first formulated the DMVT. Kijewski applied the DMVT to $\mathrm{C}^{2+}$ and found that the $G$ condition was a rather strong condition. ${ }^{10}$ Garrod et al. ${ }^{11,12}$ also implemented their method and calculated the ground state of $\mathrm{Be}$ atom very accurately. Erdahl proposed to use the convex program for solving the DMVT and performed accurate calculation for the $\mathrm{He}_{2}$ molecule. ${ }^{13}$ Afterwards, the interest for solving 2-RDM using the DMVT has almost disappeared for about 20 years. The reasons were probably that there was no rigorous mathematical and computational algorithm for the DMVT calculation, and the computer facilities were not so

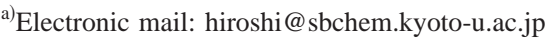

powerful at that time, so that their methods were applicable only to extremely small systems from the limitation in the number of variational parameters.

Mazziotti ${ }^{6}$ applied variational principle to Lipkin model employing positive semidefiniteness of the 4-RDM and the density equation. Erdahl and $\mathrm{Jin}^{14}$ considered a merit of using higher (than 2) order RDM in the DMVT for the existence of more effective $N$-representability condition for the model system of one-dimensional periodic lattice of electron pairs. They extended the work of Garrod and Percus for higher order RDMs, and gave some insights for using higher order RDM as a basic variable.

In our previous study, ${ }^{15}$ we could efficiently implement the DMVT using the semidefinite programming algorithm (SDPA $)^{16-19}$ and succeeded to calculate the 2-RDM of the ground state of different symmetry for many atoms and molecules. We transformed the DMVT to the standard type problem of SDP. We showed that the positive semidefiniteness conditions of the $P, Q,{ }^{8}$ and $G^{9}$ matrices were very strong for atoms and molecules, though they are only necessary conditions of the $N$-representability.

In the recent work of Mazziotti and Erdahl,${ }^{20}$ positive semidefinite condition of 3- and 4-RDMs were examined for solving the DMVT coupled with DET. They demonstrated its performance for a boson model of two-energy-level system with $N=10-75$. Valdemoro et al. also considered the functional reconstruction with respect to the ensemble representability conditions. ${ }^{21}$

Another promising approach was initiated by one of the authors. ${ }^{22-25}$ Since the exact $\Psi$ is an eigenfunction of the Hamiltonian that has simple structure composed of only oneand two-body operators, the $\Psi$ itself should also have a simple structure reflecting the simple structure of the Hamiltonian. Some explicit expressions of the structure of the ex- 
act wave function were given and the theories for the ground and excited states was formulated and applied to a simple model system.

In this paper, we extensively apply our DMVT to calculations of the potential energy surfaces of molecules. Previously, we applied our DET to calculations of the potential energy curves of small molecules. ${ }^{26}$ Though the results were encouraging around the equilibrium and elongated geometry, the calculation failed to converge at large internuclear distances. Here, special attentions are paid to the performance of DMVT for describing the electronic state of strongly correlated systems and the multiconfigurational systems. We also discuss the size-consistency property of the method in connection with the $N$-representability condition. We will also examine the Weinhold-Wilson inequalitie ${ }^{27-29}$ for the obtained 2-RDM and consider their possibilities as another $N$-representability conditions in our method.

\section{THEORY}

\section{A. Definitions and basic algorithm}

First and second order reduced density matrices (1-, 2-RDMs), $\gamma$ and $\Gamma$, are defined by

$$
\begin{aligned}
& \gamma_{j}^{i}=\left\langle\Psi\left|a_{i}^{\dagger} a_{j}\right| \Psi\right\rangle, \\
& \Gamma_{j_{1} j_{2}}^{i_{1} i_{2}}=\frac{1}{2}\left\langle\Psi\left|a_{i_{1}}^{\dagger} a_{i_{2}}^{\dagger} a_{j_{2}} a_{j_{1}}\right| \Psi\right\rangle,
\end{aligned}
$$

where $a^{\dagger}$ and $a$ are creation and annihilation operators, respectively. Practical complete $N$-representability condition is not known for 2-RDM: we know only some necessary conditions. In the present DMVT, we use $P, Q$, and $G$ conditions. The $P, Q$, and $G$ matrices are defined by

$$
\begin{aligned}
& P_{j_{1} j_{2}}^{i_{1} i_{2}}=\left\langle\Psi\left|a_{i_{1}}^{\dagger} a_{i_{2}}^{\dagger} a_{j_{2}} a_{j_{1}}\right| \Psi\right\rangle, \\
& Q_{j_{1} j_{2}}^{i_{1} i_{2}}=\left\langle\Psi\left|a_{i_{1}} a_{i_{2}} a_{j_{2}}^{\dagger} a_{j_{1}}^{\dagger}\right| \Psi\right\rangle, \\
& G_{j_{1} j_{2}}^{i_{1} i_{2}}=\left\langle\Psi\left|a_{i_{1}}^{\dagger} a_{i_{2}} a_{j_{2}}^{\dagger} a_{j_{1}}\right| \Psi\right\rangle,
\end{aligned}
$$

respectively. We enforce all of these matrices to be positive semidefinite. We also use seven trivial conditions of 2-RDM, which are antisymmetric condition, hermiticity, trace condition, number of electrons, number of spins, and expectation values of $S_{z}$ and $S^{2}$.

In the DMVT, we take 2-RDM as a variational variable, and minimize the energy within $\mathrm{N}$-representability conditions, namely,

$$
E_{\min }=\underset{\Gamma \in \mathcal{P}^{(2)}}{\operatorname{Min}} \operatorname{Tr} H \Gamma,
$$

where $H$ is the Hamiltonian of the system, $\mathcal{P}^{(2)}$ is a set of 2-RDM that satisfy approximate or nearly complete $\mathrm{N}$-representability condition. We did two types of calculations using the approximate $N$-representability conditions: one is with the trivial representability condition plus $P$ and $Q$ condition, denoted as $\operatorname{DMVT}(P Q)$, and the other is with the trivial condition plus $P, Q$, and $G$ conditions, denoted as $\operatorname{DMVT}(P Q G)$.

For implementing the minimization problem with these linear and semidefiniteness conditions, we casted this prob-

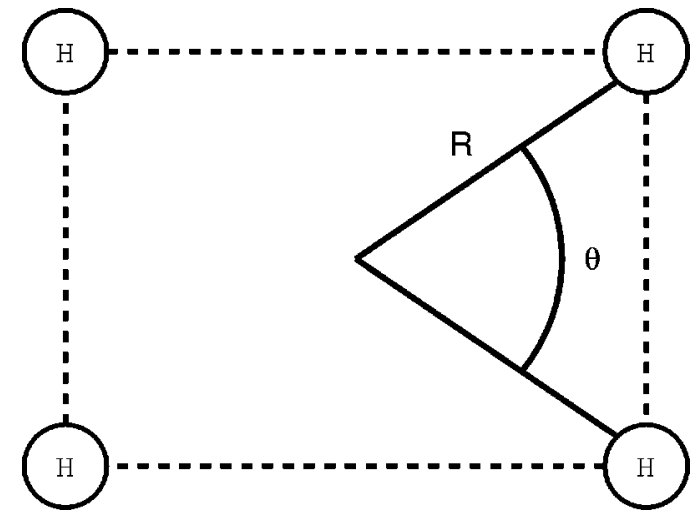

FIG. 1. Coordinates for $\mathrm{H}_{4}$.

lem into the SDP, ${ }^{16-18}$ and we employ SDPA $^{19}$ as a standard SDP solver. Details were described in Ref. 15.

\section{B. Additional linear inequalities for the density matrices}

Weinhold and Wilson, ${ }^{27}$ Davidson, ${ }^{28}$ and McRae and Davidson ${ }^{29}$ derived some other $N$-representability conditions that were expressed as linear inequalities using only the diagonal elements of 2-RDM. Among them, the conditions independent from those already used in our present method are as follows.

Condition VI:

$\gamma_{i}^{i}-2 \Gamma_{i j}^{i j}-2 \Gamma_{i k}^{i k}+2 \Gamma_{j k}^{j k} \geqslant 0$.

Condition VII:

$1-\gamma_{i}^{i}-\gamma_{j}^{j}-\gamma_{k}^{k}+2 \Gamma_{i j}^{i j}+2 \Gamma_{i k}^{i k}+2 \Gamma_{j k}^{j k} \geqslant 0$.

Condition VIII: Positive semidefiniteness of the $\Omega$ matrix

$$
\Omega=\left(\begin{array}{cccccc}
\gamma_{1}^{1} & 2 \Gamma_{12}^{12} & 2 \Gamma_{13}^{13} & \cdots & 2 \Gamma_{1 t}^{1 t} & \gamma_{1}^{1} \\
2 \Gamma_{12}^{12} & \gamma_{2}^{2} & 2 \Gamma_{23}^{23} & \cdots & 2 \Gamma_{2 t}^{2 t} & \gamma_{2}^{2} \\
2 \Gamma_{12}^{12} & 2 \Gamma_{13}^{13} & \gamma_{3}^{3} & \cdots & 2 \Gamma_{3 t}^{3 t} & \gamma_{3}^{3} \\
\vdots & \vdots & \vdots & & \vdots & \vdots \\
2 \Gamma_{1 t}^{1 t} & 2 \Gamma_{2 t}^{2 t} & 2 \Gamma_{3 t}^{3 t} & \cdots & \gamma_{t}^{t} & \gamma_{t}^{t} \\
\gamma_{1}^{1} & \gamma_{2}^{2} & \gamma_{3}^{3} & \cdots & \gamma_{t}^{t} & 1
\end{array}\right)
$$

These are the representability conditions that may be stronger than and/or may reinforce the $P, Q$, and $G$ conditions. Since these conditions are given as linear inequalities, it is easy to include them into the present DMVT formalism within the SDP formalism, since SDP is an extension of the linear programming. In this study, we examine the resultant 2-RDM against these three inequalities, the condition VI, VII, and VIII, and discuss the possibility of using these conditions as the additional constraints in our DMVT formalism.

\section{Size-consistency}

The positive semidefiniteness of the $G$ matrix includes a necessary condition for size consistency. In the original nonlinear form, the position representation of the $G$ matrix is given by 


$$
\begin{aligned}
G\left(12 \mid 1^{\prime} 2^{\prime}\right)= & \left\langle\left(\psi^{\dagger}(2) \psi(1)-\left\langle\psi^{\dagger}(2) \psi(1)\right\rangle\right)^{\dagger}\left(\psi^{\dagger}\left(2^{\prime}\right) \psi\left(1^{\prime}\right)-\left\langle\psi^{\dagger}\left(2^{\prime}\right) \psi\left(1^{\prime}\right)\right\rangle\right)\right\rangle \\
= & \left\langle\left(\psi^{\dagger}(1) \psi(2)-\left\langle\psi^{\dagger}(2) \psi(1)\right\rangle^{*}\right)\left(\psi^{\dagger}\left(2^{\prime}\right) \psi\left(1^{\prime}\right)-\left\langle\psi^{\dagger}\left(2^{\prime}\right) \psi\left(1^{\prime}\right)\right\rangle\right)\right\rangle \\
= & \left\langle\psi^{\dagger}(1) \psi(2) \psi^{\dagger}\left(2^{\prime}\right) \psi\left(1^{\prime}\right)\right\rangle-\left\langle\psi^{\dagger}(2) \psi(1)\right\rangle^{*}\left\langle\psi^{\dagger}\left(2^{\prime}\right) \psi\left(1^{\prime}\right)\right\rangle \\
& -\left\langle\psi^{\dagger}(1) \psi(2)\right\rangle\left\langle\psi^{\dagger}\left(2^{\prime}\right) \psi\left(1^{\prime}\right)\right\rangle+\left\langle\psi^{\dagger}(2) \psi(1)\right\rangle^{*}\left\langle\psi^{\dagger}\left(2^{\prime}\right) \psi\left(1^{\prime}\right)\right\rangle \\
= & \left\langle\psi^{\dagger}(1) \psi(2) \psi^{\dagger}\left(2^{\prime}\right) \psi\left(1^{\prime}\right)\right\rangle-\left\langle\psi^{\dagger}(1) \psi(2)\right\rangle\left\langle\psi^{\dagger}\left(2^{\prime}\right) \psi\left(1^{\prime}\right)\right\rangle,
\end{aligned}
$$

where $\psi(i)$ is a field operator defined by using one-particle complete basis set $\left\{\psi_{j}\right\}$,

$$
\psi(i)=\sum_{j} \psi_{j}(i) a_{j} .
$$

A static density-density autocorrelation function $F\left(1 \mid 1^{\prime}\right)^{30}$ corresponds to the $G$ matrix as

$$
\begin{aligned}
F\left(1 \mid 1^{\prime}\right)= & \left\langle n(1) n\left(1^{\prime}\right)\right\rangle-\langle n(1)\rangle\left\langle n\left(1^{\prime}\right)\right\rangle \\
= & \left\langle\psi^{\dagger}(1) \psi(1) \psi^{\dagger}\left(1^{\prime}\right) \psi\left(1^{\prime}\right)\right\rangle \\
& -\left\langle\psi^{\dagger}(1) \psi(1)\right\rangle\left\langle\psi^{\dagger}\left(1^{\prime}\right) \psi\left(1^{\prime}\right)\right\rangle \\
= & G\left(11 \mid 1^{\prime} 1^{\prime}\right),
\end{aligned}
$$

where $n(i)$ is the density operator defined by

$$
n(i)=\psi^{\dagger}(i) \psi(i) \text {. }
$$

Using the positive semidefiniteness of $G\left(12 \mid 1^{\prime} 2^{\prime}\right)$,

$$
\int x(12) G\left(12 \mid 1^{\prime} 2^{\prime}\right) x\left(1^{\prime} 2^{\prime}\right)^{*} d \tau_{1} d \tau_{2} d \tau_{1^{\prime}} d \tau_{2^{\prime}} \geqslant 0,
$$

where $x(12)$ is an arbitrary two particle function, $F\left(1 \mid 1^{\prime}\right)$ is shown to be also positive semidefinite by integrating the $G$ matrix with respect to the two particle function $x(12)$ given by $x(12)=x(1) \delta(1-2)$, as

$$
\begin{aligned}
0 \leqslant & \int x(12) G\left(12 \mid 1^{\prime} 2^{\prime}\right) x\left(1^{\prime} 2^{\prime}\right)^{*} d \tau_{1} d \tau_{2} d \tau_{1^{\prime}} d \tau_{2^{\prime}} \\
= & \int x(1) \delta(1-2) G\left(12 \mid 1^{\prime} 2^{\prime}\right) x\left(1^{\prime}\right)^{*} \delta\left(1^{\prime}-2^{\prime}\right)^{*} \\
& \times d \tau_{1} d \tau_{2} d \tau_{1^{\prime}} d \tau_{2^{\prime}} \\
= & \int x(1) G\left(11 \mid 1^{\prime} 1^{\prime}\right) x\left(1^{\prime}\right)^{*} d \tau_{1} d \tau_{1^{\prime}} \\
= & \int x(1) F\left(1 \mid 1^{\prime}\right) x\left(1^{\prime}\right)^{*} d \tau_{1} d \tau_{1^{\prime}}
\end{aligned}
$$

TABLE I. Total energy and correlation energy in $(\%)$ for $\mathrm{H}_{4}$ as a function of $R$ with $\theta$ fixed at 90 degree.

\begin{tabular}{lcccc}
\hline \hline$R(\AA)$ & $\operatorname{DMVT}(P Q)$ & $\operatorname{DMVT}(P Q G)$ & Full-CI & Hartree-Fock \\
\hline 0.6 & $-2.0405(186)$ & $-1.9553(104)$ & $-1.9511(100)$ & $-1.8474(0)$ \\
0.8 & $-2.1485(168)$ & $-2.0629(101)$ & $-2.0610(100)$ & $-1.9330(0)$ \\
1.0 & $-2.1881(177)$ & $-2.0693(101)$ & $-2.0684(100)$ & $-1.9122(0)$ \\
1.2 & $-2.2210(191)$ & $-2.0480(100)$ & $-2.0474(100)$ & $-1.8568(0)$ \\
1.4 & $-2.2407(194)$ & $-2.0251(100)$ & $-2.0246(100)$ & $-1.7939(0)$ \\
1.6 & $-2.2367(183)$ & $-2.0087(100)$ & $-2.0085(100)$ & $-1.7340(0)$ \\
1.8 & $-2.2226(141)$ & $-1.9993(100)$ & $-1.9992(100)$ & $-1.4551(0)$ \\
2.0 & $-2.2055(141)$ & $-1.9945(100)$ & $-1.9945(100)$ & $-1.4818(0)$ \\
\hline \hline
\end{tabular}

where $x(1)$ is an arbitrary one-particle function. From the positive semidefiniteness of $F\left(1 \mid 1^{\prime}\right)$, it is shown that $F\left(1 \mid 1^{\prime}\right)$ is everywhere non-negative.

The size consistency requires more strict condition; when $\left|1-1^{\prime}\right| \rightarrow \infty, F\left(1 \mid 1^{\prime}\right)$ should asymptotically go to zero, namely,

$$
\lim _{\left|1-1^{\prime}\right| \rightarrow \infty} F\left(1 \mid 1^{\prime}\right)=0 .
$$

The positive semidefiniteness of the $G$ matrix guarantees only the non-negativity of $F\left(1 \mid 1^{\prime}\right)$, but does not guarantee this asymptotical condition. Thus, the $\operatorname{DMVT}(P Q G)$ includes a necessary condition for the size-consistency, while in the $\operatorname{DMVT}(P Q)$, even $F\left(1 \mid 1^{\prime}\right)$ is not necessarily nonnegative.

\section{RESULTS AND DISCUSSIONS}

\section{A. $\mathrm{H}_{4}$ system}

First, we applied our DMVT to the potential energy surface of $\mathrm{H}_{4}$. This system has been frequently used as a benchmark molecule of many methods for the quasidegenerate situation: $^{31,32}$ the $a_{g} b_{2 u}$ and $a_{g} b_{3 u}$ configurations become equivalent for a square geometry and therefore, become degenerate. We used the DZ basis set ${ }^{33,34}$ for $\mathrm{H}$ and defined the potential energy surface with the coordinates $(\theta, R)$ depicted in Fig. 1. $R$ gives the size of the molecule and $\theta$ defines the asymmetry of the structure. We calculated three different cuts of the potential energy surface that were also tested in Ref. 31.

First, we examined the cut of stretching $R$ with $\theta=90^{\circ}$ fixed, namely, the square structure as a function of $R$. The results are summarized in Table I. The DMVT $(P Q G)$ reproduced the full-CI curve quite accurately. For large $R$, it gave almost identical total energy and the errors were within 1 mhartree for $R>1.0 \AA$, though the total correlation energies

TABLE II. Total energy and correlation energy in $(\%)$ for $\mathrm{H}_{4}$ as a function of $\theta$ with $R$ fixed at the equilibrium value of $0.869 \AA$.

\begin{tabular}{ccccc}
\hline \hline$\theta$ (degrees) & DMVT $(P Q)$ & DMVT $(P Q G)$ & Full-CI & Hartree-Fock \\
\hline 90.0 & $-2.1656(170)$ & $-2.0711(101)$ & $-2.0697(100)$ & $-1.9326(0)$ \\
89.9 & $-2.1656(170)$ & $-2.0711(101)$ & $-2.0697(100)$ & $-1.9335(0)$ \\
89.5 & $-2.1656(172)$ & $-2.0713(101)$ & $-2.0698(100)$ & $-1.9372(0)$ \\
89.0 & $-2.1655(174)$ & $-2.0718(101)$ & $-2.0703(100)$ & $-1.9418(0)$ \\
88.0 & $-2.1654(177)$ & $-2.0738(101)$ & $-2.0721(100)$ & $-1.9509(0)$ \\
85.0 & $-2.1673(180)$ & $-2.0849(102)$ & $-2.0830(100)$ & $-1.9777(0)$ \\
80.0 & $-2.1869(185)$ & $-2.1120(101)$ & $-2.1106(100)$ & $-2.0205(0)$ \\
70.0 & $-2.2337(185)$ & $-2.1727(101)$ & $-2.1721(100)$ & $-2.0992(0)$ \\
\hline \hline
\end{tabular}


TABLE III. Total energy and correlation energy in (\%) for $\mathrm{H}_{4}$ as a function of $\theta$ with $R$ fixed at $1.738 \AA$.

\begin{tabular}{ccccc}
\hline \hline$\theta$ (degrees) & DMVT $(P Q)$ & DMVT $(P Q G)$ & Full-CI & Hartree-Fock \\
\hline 90.0 & $-2.2275(174)$ & $-2.0016(100)$ & $-2.0015(100)$ & $-1.6962(0)$ \\
89.9 & $-2.2275(174)$ & $-2.0015(100)$ & $-2.0015(100)$ & $-1.6967(0)$ \\
89.5 & $-2.2276(175)$ & $-2.0017(100)$ & $-2.0015(100)$ & $-1.6988(0)$ \\
89.0 & $-2.2276(175)$ & $-2.0019(100)$ & $-2.0015(100)$ & $-1.7014(0)$ \\
88.0 & $-2.2278(177)$ & $-2.0023(100)$ & $-2.0018(100)$ & $-1.7067(0)$ \\
85.0 & $-2.2289(181)$ & $-2.0041(100)$ & $-2.0033(100)$ & $-1.7231(0)$ \\
80.0 & $-2.2327(188)$ & $-2.0087(100)$ & $-2.0080(100)$ & $-1.7523(0)$ \\
70.0 & $-2.2465(207)$ & $-2.0258(100)$ & $-2.0255(100)$ & $-1.8198(0)$ \\
\hline \hline
\end{tabular}

were large, for example, 0.51 a.u. for $R=2.0 \AA$. The method was found to give a good description for the quasidegenerate system. On the other hand, the DMVT $(P Q)$ gave $40-90 \%$ errors of the correlation energies.

Second, the cut of $\theta$ ranging from $70.0^{\circ}$ to $90.0^{\circ}$ with $R=0.869 \AA$, which is near equilibrium distance is examined in Table II. At $\theta=90^{\circ}$, electronic state becomes quasidegenerate. The $\operatorname{DMVT}(P Q G)$ gave very smooth potential curve parallel to the full-CI without artificial cusp at $\theta=90^{\circ} .{ }^{31}$ The deviations were within 2 mhartree and $2 \%$ of the total correlation energy throughout the geometries. For this system, the errors were constant regardless of the quasidegeneracy.

Last, the cut of $\theta=70^{\circ}-90^{\circ}$ with $R$ elongated to 1.738 $\AA$, namely, $2 \times R_{e}$, is examined in Table III. Surprisingly, the $\operatorname{DMVT}(P Q G)$ gave almost identical results with the full-CI ones: the deviations were less than 1 mhartree for all the geometries. Though the present calculations did not include polarization functions, the DMVT $(P Q G)$ gave very accurate potential energy surface of $\mathrm{H}_{4}$.

For this system all the Weinhold-Wilson inequalities were satisfied for all the potential energy surfaces examined here. This also supports the high quality of 2-RDM calculated by the $\operatorname{DMVT}(P Q G)$.

\section{B. Ne and the equilibrium geometry of $\mathrm{N}_{2}, \mathrm{CO}, \mathrm{C}_{2}$, $\mathrm{LiF}$, and $\mathrm{CH}_{4}$}

Next, the DMVT is applied to the ground state of Ne, $\mathrm{N}_{2}, \mathrm{CO}, \mathrm{C}_{2}, \mathrm{LiF}$, and $\mathrm{CH}_{4}$, which were not calculated in the previous study. ${ }^{15}$ In Table IV, we summarized the total energy for these systems. In all calculations, we adopted STO-6G minimal basis ${ }^{35}$ and experimental geometries ${ }^{36,37}$ except for Ne. For Ne, $[3 s 2 p]$ basis set was used. The $1 s$ orbitals of the second raw atoms were fixed as cores.
Generally, the results of $\operatorname{DMVT}(P Q G)$ calculations were satisfactory except for $\mathrm{C}_{2}$ and $\mathrm{CH}_{4}$. The $\operatorname{DMVT}(P Q)$ calculations overshoot the energy of these molecules, especially for $\mathrm{C}_{2}$, by $802 \%$. The DMVT $(P Q G)$ recovered it up to $117 \%$. The deviation is still not small, however, the convergence to the exact value is encouraging since the ground state of $\mathrm{C}_{2}$ is known to be quasidegenerate even at the equilibrium geometry. We also obtained remarkable improvement for other systems by requiring the $G$ condition.

The Weinhold-Wilson inequalities VI, VII, and VIII were examined for these systems. For $\mathrm{CO}, \mathrm{LiF}$, and $\mathrm{Ne}$, all of the inequalities were satisfied. For $\mathrm{CH}_{4}$ and $\mathrm{C}_{2}$, the inequality type VI and VII were violated, and for $\mathrm{N}_{2}$, the inequality type VI was violated but others were satisfied, though the violations were very small as $-0.000297--0.002063$ $\left(\mathrm{CH}_{4}\right.$, type $\left.\mathrm{VI}\right),-0.000127-0.001803\left(\mathrm{CH}_{4}\right.$, type $\left.\mathrm{VII}\right)$, $-0.004169--0.019446\left(\mathrm{C}_{2}\right.$, type $\left.\mathrm{VI}\right), \quad-0.000169-$ $-0.030852\left(\mathrm{C}_{2}\right.$, type VII) and $-0.00283--0.00551$ $\left(\mathrm{N}_{2}\right.$, type $\left.\mathrm{VI}\right)$, respectively. These violations were parallel to the errors of the $\operatorname{DMVT}(P Q G)$ calculations: the deviations $\mathrm{CH}_{4}(124 \%), \mathrm{C}_{2}(117 \%)$, and $\mathrm{N}_{2}(108 \%)$ were larger than those of the other systems. This implies that the inequalities VI and VII may be adopted as one of the additional $\mathrm{N}$-representability conditions for the DMVT.

\section{Potential curves of $\mathrm{H}_{2} \mathrm{O}, \mathrm{NH}_{3}$, and $\mathrm{BH}_{3}$}

Double dissociation of $\mathrm{H}_{2} \mathrm{O}$ and triple dissociation of $\mathrm{NH}_{3}$ and $\mathrm{BH}_{3}$ are interesting examples, since four and six electrons are correlated in the bond dissociation processes. We calculated the potential curves for the symmetric stretching mode of these systems at several points within $R$ $=0.5-5.0 \AA$, and the results were shown in Figs. 2-4. We used STO-6G basis set and kept $1 s$ orbitals of $\mathrm{O}, \mathrm{N}$, and B to be frozen. Spectroscopic constants of equilibrium distance $\left(r_{e}\right)$, harmonic frequency $\left(\omega_{e}\right)$, and dissociation energy $\left(D_{e}\right)$ were summarized in Table V. The potential energy curve was fit with the 6th extended Rydberg function for some points near the equilibrium geometry and the $\omega_{e}$ was calculated by the Dunhum method. ${ }^{38}$ The $\mathrm{H}-\mathrm{O}-\mathrm{H}$ and $\mathrm{H}-\mathrm{N}-\mathrm{H}$ angles were fixed at the experimental values and only the $\mathrm{H}-\mathrm{O}$ and $\mathrm{H}-\mathrm{N}$ bonds were symmetrically stretched: $\omega_{e}$ was defined for this coordinate and therefore different from that of the normal mode analysis.

For $\mathrm{H}_{2} \mathrm{O}$ and $\mathrm{NH}_{3}$, DMVT $(P Q G)$ simulated the full-CI curves very accurately even up to the dissociation limit and the two curves almost overlapped. $\mathrm{H}_{2} \mathrm{O}$ and $\mathrm{NH}_{3}$ dissociate

TABLE IV. Total energy and correlation energy in $(\%) \mathrm{Ne}, \mathrm{CO}, \mathrm{N}_{2}, \mathrm{LiF}, \mathrm{C}_{2}$, and $\mathrm{CH}_{4}$ at equilibrium geometry.

\begin{tabular}{lcccccrc}
\hline \hline System & State & MO $^{\mathrm{a}}$ & Act. Ele $^{\mathrm{b}}$ & DMVT $(P Q)$ & DMVT $(P Q G)$ & Full-CI & Hartree-Fock \\
\hline $\mathrm{Ne}$ & ${ }^{1} S$ & $8(9)$ & 8 & $-129.2430(705)$ & $-128.6292(105)$ & $-128.6245(100)$ & $-128.5224(0)$ \\
$\mathrm{CO}$ & ${ }^{1} \Sigma$ & $8(10)$ & 12 & $-113.1163(584)$ & $-112.4544(108)$ & $-112.4426(100)$ & $-112.3033(0)$ \\
$\mathrm{N}_{2}$ & ${ }^{1} \Sigma_{\mathrm{g}}^{+}$ & $8(10)$ & 12 & $-109.4466(571)$ & $-108.7123(108)$ & $-108.7002(100)$ & $-108.5418(0)$ \\
$\mathrm{LiF}$ & ${ }^{1} \mathrm{C}_{2}$ & $8(10)$ & 10 & $-106.7727(568)$ & $-106.4448(102)$ & $-106.4435(100)$ & $-106.3731(0)$ \\
$\mathrm{C}_{2}$ & ${ }^{1} \Sigma_{\mathrm{g}}^{+}$ & $8(10)$ & 10 & $-77.3387(802)$ & $-75.4793(117)$ & $-75.4340(100)$ & $-75.1626(0)$ \\
$\mathrm{CH}_{4}$ & ${ }^{1} A_{1}$ & $8(10)$ & 8 & $-40.4335(403)$ & $-40.2100(124)$ & $-40.1905(100)$ & $-40.1102(0)$ \\
\hline \hline
\end{tabular}

${ }^{a}$ Number of active MOs, with the number of total MOs in parentheses.

${ }^{\mathrm{b}}$ Number of active electrons. 


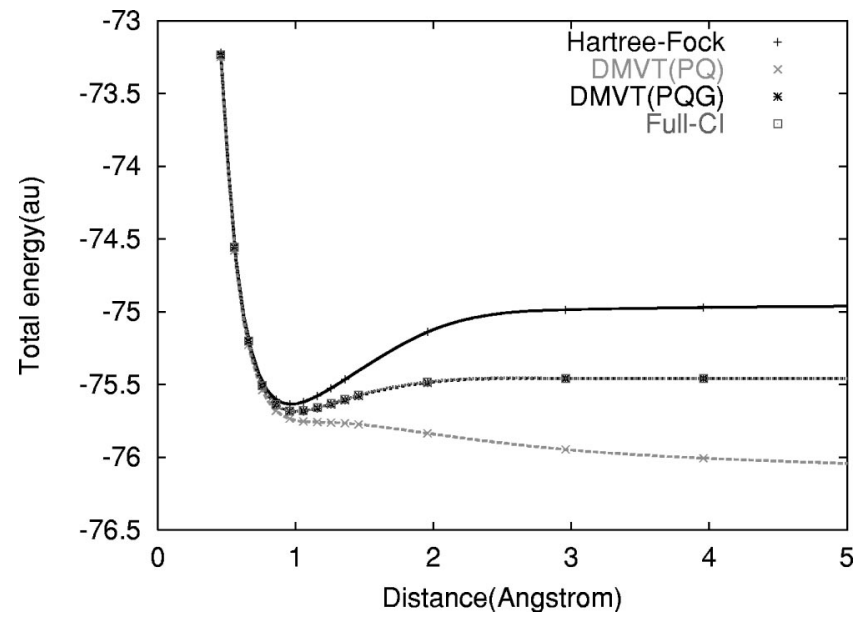

FIG. 2. Potential curve for the double dissociation of $\mathrm{H}_{2} \mathrm{O}$.

into $\mathrm{O}\left({ }^{3} P\right)+\mathrm{H}\left({ }^{1} S\right)+\mathrm{H}\left({ }^{1} S\right)$ and $\mathrm{N}\left({ }^{4} S\right)+\mathrm{H}\left({ }^{1} S\right)+\mathrm{H}\left({ }^{1} S\right)$ $+\mathrm{H}\left({ }^{1} S\right)$, respectively, and at the dissociation limit, the electronic state becomes a multiconfigurational state. $\operatorname{DMVT}(P Q G)$ accurately described these multiconfigurational states, namely, static electron correlations: the deviations from the full-CI were less than 0.5 mhartree in the dissociation limit. On the other hand, while $\operatorname{DMVT}(P Q)$ reproduced the curves in the short bond region, it failed at the large internuclear distances. DMVT $(P Q)$ curve did not bound. For $\mathrm{BH}_{3}$, even the DMVT $(P Q G)$ curve slightly deviates from the full-CI curve for $R_{\mathrm{B}-\mathrm{H}}>2.0 \AA$. The dissociation limit of $\mathrm{BH}_{3}$ is heavily quasidegenerate: the electronic state is represented by several configurations including quadruple excitations. DMVT $(P Q)$ curve for $\mathrm{BH}_{3}$ has a hump at around $1.5 \AA$, and, the potential curve is repulsive in nature.

Since DMVT $(P Q G)$ calculations gave accurate potential curves, their spectroscopic constants were also accurate. For these systems, the deviations from the full-CI were within $0.003 \AA$ and $30 \mathrm{~cm}^{-1}$, for $r_{e}$ and $\omega_{e}$, respectively. The dissociation energies $\left(D_{e}\right)$ were estimated slightly larger by $0.06,0.27$, and $0.26 \mathrm{eV}$, for $\mathrm{H}_{2} \mathrm{O}, \mathrm{NH}_{3}$ and $\mathrm{BH}_{3}$, respectively. This is because DMVT $(P Q G)$ calculations overshoot

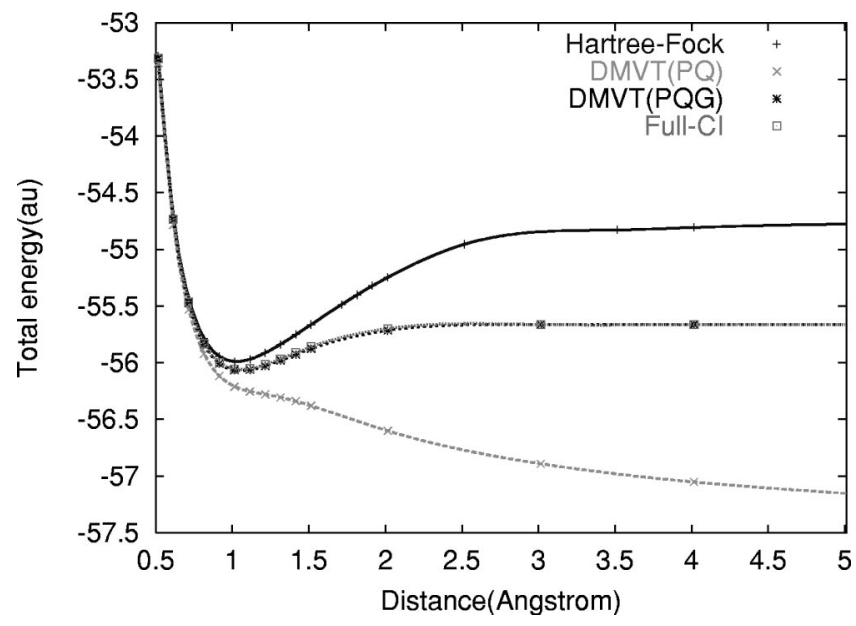

FIG. 3. Potential curve for the triple dissociation of $\mathrm{NH}_{3}$.

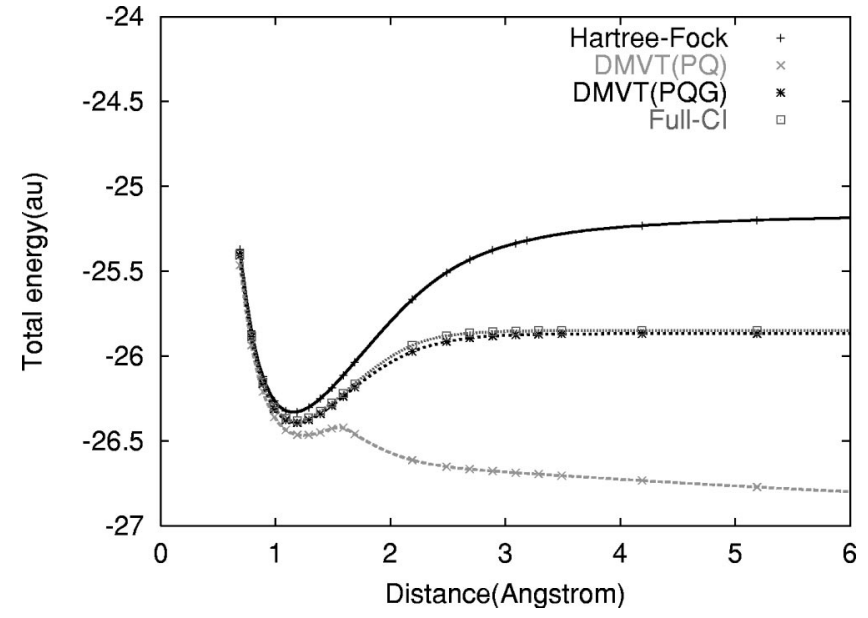

FIG. 4. Potential curve for the triple dissociation of $\mathrm{BH}_{3}$.

the full-CI energy around the equilibrium geometries rather than deviate in the dissociation limit. Since the dissociations of these systems are homolytic and include multiple bonds, the Hartree-Fock description of the dissociation limit was of course very crude.

We examined the Weinhold-Wilson inequalities for $\mathrm{BH}_{3}$, since the deviation from the full-CI was large for this molecule. Actually, the violations of the conditions VI and VII at the equilibrium distance ranged -0.000019-0.003018 and $-0.000391--0.002608$, respectively, and those of the conditions VI and VII were -0.004804-0.013975 , and $-0.001448--0.001956$ at the dissociation limit $(R=5 \AA)$.

\section{Potential curves of $\mathrm{CO}, \mathrm{C}_{2}, \mathrm{~N}_{2}$, and $\mathrm{Be}_{2}$}

Next, we apply the DMVT to the potential energy curves of $\mathrm{CO}, \mathrm{C}_{2}, \mathrm{~N}_{2}$, and $\mathrm{Be}_{2}$, since their electronic states are

TABLE V. Spectroscopic constants of $\mathrm{H}_{2} \mathrm{O}, \mathrm{NH}_{3}, \mathrm{BH}_{3}, \mathrm{C}_{2}, \mathrm{~N}_{2}$, and CO.

\begin{tabular}{llccr}
\hline \hline System & \multicolumn{1}{c}{ Method } & $r_{e}(\AA)$ & $\omega_{e}\left(\mathrm{~cm}^{-1}\right)$ & $D_{e}(\mathrm{eV})$ \\
\hline $\mathrm{H}_{2} \mathrm{O}$ & Hartree-Fock & 1.824 & 3952 & 18.471 \\
& Full-CI & 1.895 & 3253 & 6.162 \\
& DMVT $(P Q G)$ & 1.894 & 3276 & 6.227 \\
$\mathrm{NH}_{3}$ & Hartree-Fock & 1.025 & 3750 & 33.008 \\
& Full-CI & 1.057 & 3324 & 10.686 \\
& DMVT $(P Q G)$ & 1.057 & 3291 & 10.956 \\
$\mathrm{BH}_{3}$ & Hartree-Fock & 1.154 & 3115 & 31.284 \\
& Full-CI & 1.178 & 2883 & 14.280 \\
& DMVT $(P Q G)$ & 1.181 & 2854 & 14.537 \\
$\mathrm{C}_{2}$ & Hartree-Fock & 1.233 & 2207 & 16.876 \\
& Full-CI & 1.257 & 2035 & 6.790 \\
& DMVT $(P Q G)$ & 1.299 & 1679 & 7.212 \\
$\mathrm{~N}_{2}$ & Hartree-Fock & 1.129 & 2715 & 31.211 \\
& Full-CI & 1.210 & 2061 & 6.220 \\
& DMVT $(P Q G)$ & 1.199 & 1980 & 6.622 \\
$\mathrm{CO}$ & Hartree-Fock & 1.146 & 2461 & 12.692 \\
& Full-CI & 1.193 & 2063 & 9.328 \\
& DMVT $(P Q G)$ & 1.201 & 1990 & 9.540 \\
\hline \hline
\end{tabular}




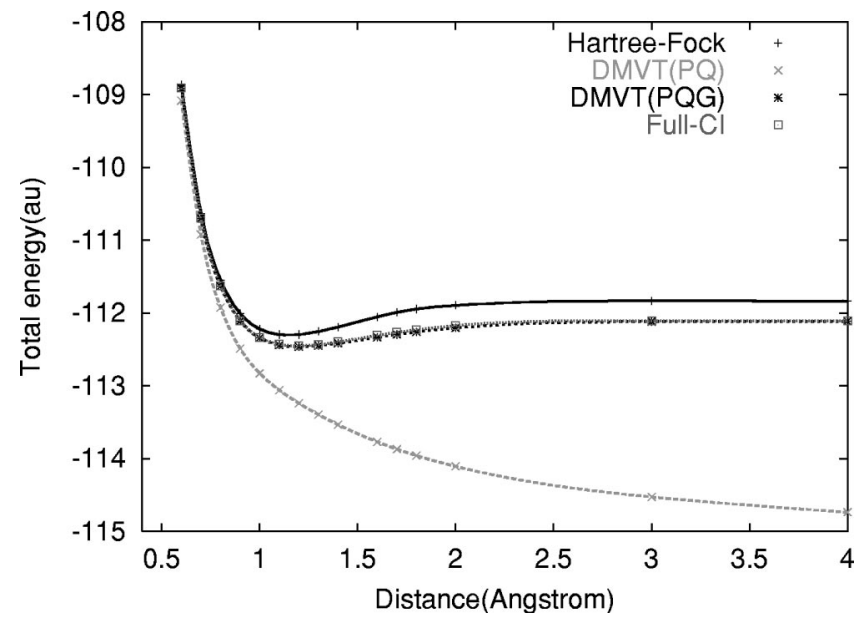

FIG. 5. Potential curve of CO.

very characteristic. In the potential curve of $\mathrm{CO}$, the Hartree-Fock configuration is dominant at around the equilibrium distance, but its weight decreases as the distance increases and finally becomes zero at the dissociation limit. $\mathrm{C}_{2}$ has unoccupied $p \sigma \mathrm{MO}$, therefore, the ground state is always quasidegenerate even in the equilibrium geometry. $\mathrm{N}_{2}$ includes triple-bond dissociation, therefore its potential curve is highly quasidegenerate at large internuclear distance. $\mathrm{Be}_{2}$ has no bonding interaction. Potential curves of these molecules were calculated for $R=0.5-5.0 \AA$. Minimal STO-6G basis set was used and the $1 s$ orbitals were kept as frozen. The potential curves were shown in Figs. 5-8 and the spectroscopic constants were given in Table $\mathrm{V}$.

As in other systems, DMVT $(P Q G)$ curves almost overlapped with the full-CI curves, while $\operatorname{DMVT}(P Q)$ curves were calculated as repulsive. The deviations of $\operatorname{DMVT}(P Q G)$ from the full-CI increases in the order of $\mathrm{N}_{2}$, $\mathrm{CO}$, and $\mathrm{C}_{2}$. Though it is true that the description of the quasidegeneracy of $\mathrm{C}_{2}$ is difficult, there is another factor in the accuracy. Since we used minimal basis set, the calculations of $\mathrm{N}_{2}$ and $\mathrm{CO}$ were for 16 spin orbitals with 12 electrons, namely, 4 hole spin orbitals, while those of $\mathrm{C}_{2}$ are for 16 spin orbitals with 10 electrons; 6 hole spin orbitals. We

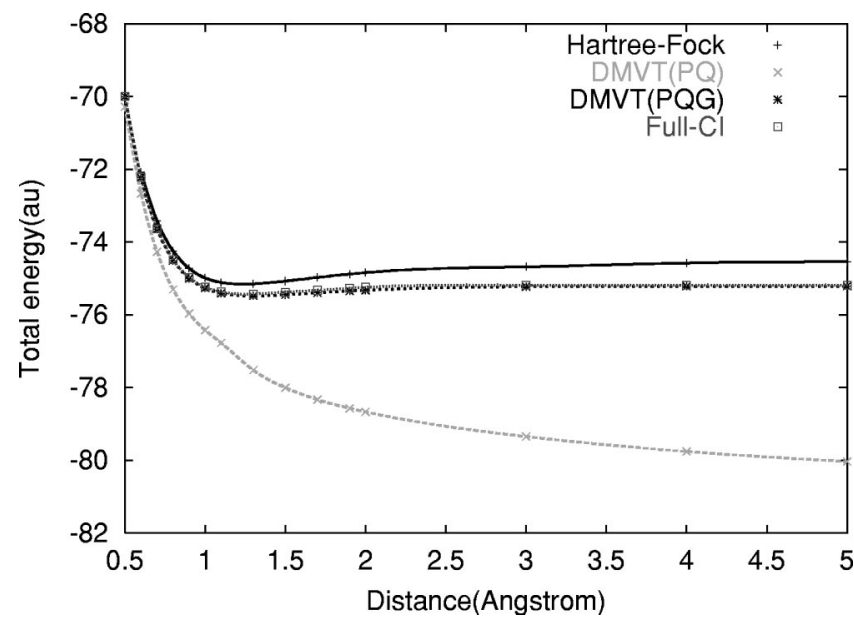

FIG. 6. Potential curve of $\mathrm{C}_{2}$.

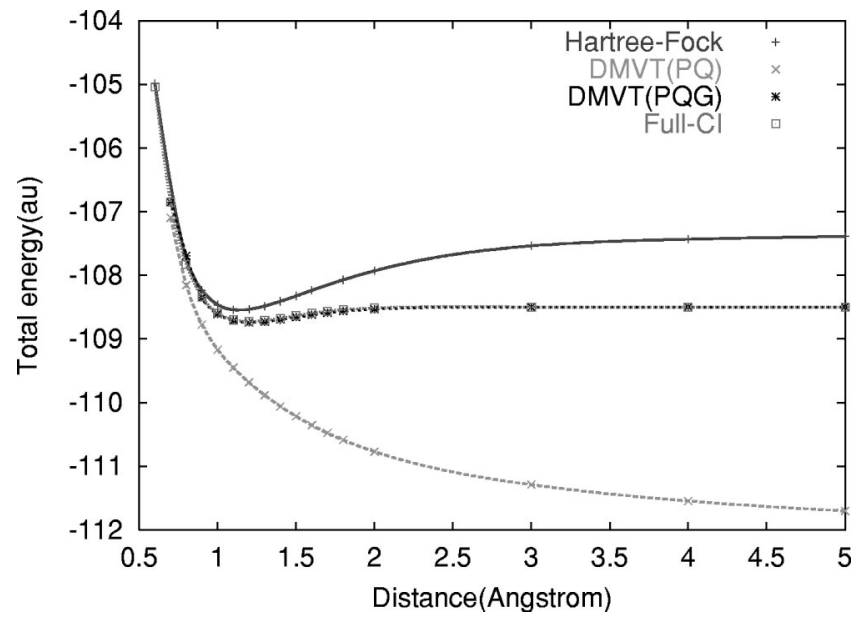

FIG. 7. Potential curve of $\mathrm{N}_{2}$.

think this also affected the accuracy of the results. The potential curve of $\mathrm{Be}_{2}$ was repulsive, since van der Waals interaction was not described by the present basis set. The $\operatorname{DMVT}(P Q)$ gave better description than other systems.

The DMVT $(P Q G)$ results for the spectroscopic constants of these diatomic molecules were less accurate than those for $\mathrm{H}_{2} \mathrm{O}$ and $\mathrm{NH}_{3}$. The deviations were $\sim 0.01 \AA$ and $\sim 80 \mathrm{~cm}^{-1}$ for $r_{e}$ and $\omega_{e}$, respectively, for $\mathrm{CO}$ and $\mathrm{N}_{2}$. For $\mathrm{C}_{2}$, the errors were as large as $0.04 \AA$ and $350 \mathrm{~cm}^{-1}$. These results reflect the quality of the DMVT around the equilibrium geometry.

We also calculated the Weinhold-Wilson inequalities for $\mathrm{C}_{2}$ and $\mathrm{CO}$. As expected, large violations occurred for the inequalities VI and VII. For $\mathrm{C}_{2}$, the violations were calculated as $-0.001810--0.027667$ and $-0.000900-$ -0.011494 for conditions VI and VII, respectively, at $R$ $=1.5 \AA$, and $-0.003047--0.002500$ only for condition VI at $R=5.0 \AA$ : the violations at $R=1.5 \AA$ were larger than those at $R=5.0 \AA$. The ground state of $\mathrm{C}_{2}$ is quasidegenerate even at the equilibrium geometry and this is the reason of the crude spectroscopic constants for $\mathrm{C}_{2}$ by the $\operatorname{DMVT}(P Q G)$. For $\mathrm{CO}$, the violations ranged $-0.008185--0.008434$ for condition VI at the dissociation

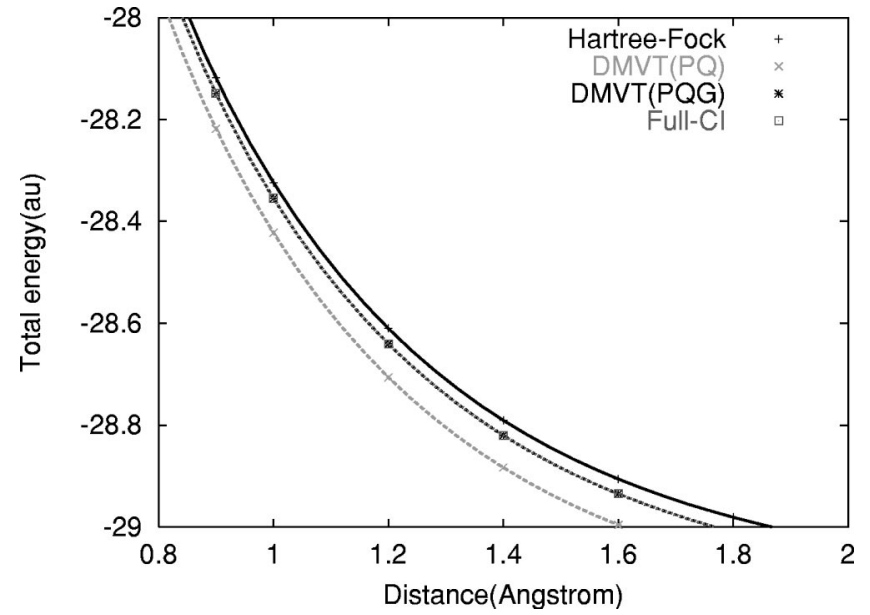

FIG. 8. Potential curve of $\mathrm{Be}_{2}$. 
TABLE VI. Examination of size consistency for $\mathrm{Ne}$ and $\mathrm{H}_{2} \mathrm{O}, \mathrm{NH}_{3}, \mathrm{BH}_{3}$, $\mathrm{Be}_{2}, \mathrm{CO}$, and $\mathrm{C}_{2}$.

\begin{tabular}{llccc}
\hline \hline System & \multicolumn{1}{c}{ Method } & $E_{\text {mol }}{ }^{\mathrm{a}}$ & $E_{\text {atom }}{ }^{\mathrm{b}}$ & $\Delta E$ \\
\hline $\mathrm{H}_{2} \mathrm{O}$ & DMVT $(P Q G)$ & -75.4589 & -75.4589 & 0.0000 \\
& Full-CI & -75.4588 & -75.4588 & 0.0000 \\
$\mathrm{NH}_{3}$ & DMVT $(P Q G)$ & -55.6622 & -55.6622 & 0.0000 \\
& Full-CI & -55.6622 & -55.6622 & 0.0000 \\
$\mathrm{BH}_{3}$ & DMVT $(P Q G)$ & -25.8680 & -25.8482 & 0.0198 \\
& Full-CI & -25.8482 & -25.8482 & 0.0000 \\
$\mathrm{Be}_{2}$ & DMVT $(P Q G)$ & -29.1655 & -29.1654 & 0.0001 \\
& Full-CI & -29.1654 & -29.1654 & 0.0000 \\
$\mathrm{CO}$ & DMVT $(P Q G)$ & -112.1153 & -112.1095 & 0.0058 \\
& Full-CI & -112.1095 & -112.1095 & 0.0000 \\
$\mathrm{C}_{2}$ & DMVT(PQG) & -75.2187 & -75.1854 & 0.0333 \\
& Full-CI & -75.1854 & -75.1854 & 0.0000 \\
$\mathrm{~N}_{2}$ & DMVT $(P Q G)$ & -108.4982 & -108.4982 & 0.0000 \\
& Full-CI & -108.4982 & -108.4982 & 0.0000 \\
\hline \hline
\end{tabular}

${ }^{a}$ Energy of molecule at the dissociation limit.

${ }^{\mathrm{b}} \mathrm{Sum}$ of the energies of the isolated atoms.

limit and -0.000546 for condition VI at $R=1.3 \AA$. There were no errors of conditions VII and VIII for CO.

\section{E. Size consistency}

As we discussed in Sec IIC, the $G$ condition is very important for the size-consistent property of the method. Here, we examine the size consistency of the results. In Table VI, the total energy of the molecule in the dissociation limit and the sum of the total energies of the isolated atoms are compared for $\mathrm{H}_{2} \mathrm{O}, \mathrm{NH}_{3}, \mathrm{BH}_{3}, \mathrm{~N}_{2}, \mathrm{C}_{2}, \mathrm{CO}$, and $\mathrm{Be}_{2}$. For $\mathrm{H}_{2} \mathrm{O}, \mathrm{NH}_{3}$, and $\mathrm{N}_{2}$, the total energies calculated by $\operatorname{DMVT}(P Q G)$ agree within numerical accuracy, which shows the size consistency holds for these systems. In these systems, Weinhold-Wilson inequalities were also satisfied and the calculations were quite accurate. As seen from the potential curves, DMVT $(P Q)$ calculations gave miserable results from the standpoint of the size consistency. The $G$ condition is apparently indispensable for the size-consistent property. For other systems, $\mathrm{BH}_{3}, \mathrm{C}_{2}, \mathrm{CO}$, and $\mathrm{Be}_{2}$, the size consistency of $\operatorname{DMVT}(P Q G)$ was not satisfactory. This is because the $G$ condition is not a sufficient condition for the size consistency. Note that the Weinhold-Wilson inequalities VI and VII were not satisfied for these systems.

\section{F. Artificially correlation enhanced system}

It is interesting to see the performance of the present method for the strongly correlated system. We here introduced the model Hamiltonian in which the electron correlations are controlled by a parameter. The Hamiltonian is partitioned into $F$, Fock operator and the rest, V:

$$
H=F+\lambda V,
$$

where $\lambda$ is a real parameter that controls the strength of the electron correlations and $\lambda=1$ corresponds to the original Hamiltonian. We adopted $\mathrm{Be}$ and $\mathrm{H}_{2} \mathrm{O}$ and changed $\lambda$ form 0.1-10000 and the results were shown in Tables VII and VIII, respectively.

For Be system, the DMVT $(P Q G)$ reproduced the exact correlation energy quite accurately and the deviations were even tempered for the variation of $\lambda$ within $1 \%$. On the other hand, the $\operatorname{DMVT}(P Q)$ gave random errors for the variation of $\lambda$. For $\mathrm{H}_{2} \mathrm{O}$, the errors of the $\operatorname{DMVT}(P Q G)$ became large, but, were within $15 \%$ relative to the total electron correlations. The DMVT $(P Q G)$ calculations converged even for the heavily correlated systems $(\lambda=10000)$, though the absolute errors were not small.

We did not see the Weinhold-Wilson violations in the Be system for all $\lambda$. The violations for $\mathrm{H}_{2} \mathrm{O}$ were not so simple. For $\lambda=1.0$, the violations of the condition VI occurred as $-0.00054--0.00368$, but no violations occurred for $\lambda=2.0$, which has the largest correlation energy error in percent. For $\lambda=10000$, we got large violations as $-0.00871--0.001036$.

\section{CONCLUSION}

The DMVT was applied to the calculations of the potential energy surfaces of the atoms and small molecules, $\mathrm{Ne}$, $\mathrm{H}_{4}, \mathrm{H}_{2} \mathrm{O}, \mathrm{NH}_{3}, \mathrm{BH}_{3}, \mathrm{CO}, \mathrm{N}_{2}, \mathrm{C}_{2}$, and $\mathrm{Be}_{2}$. This is the first study in which the bond dissociation was properly described by the DMVT. In the previous DET study of potential curves ${ }^{26}$ the results were good up to $R \sim 2 R_{e}$, but at large distances, the calculations failed to converge. Generally, the $\operatorname{DMVT}(P Q G)$ calculation reproduced the full-CI curves very accurately and they sometimes overlapped even in the dissociation limit, though the potential curves for $\mathrm{BH}_{3}$ and $\mathrm{C}_{2}$ were less accurate than others. The quasidegenerate states were well described by the DMVT $(P Q G)$ calculations. On

TABLE VII. Total energy for the model Hamiltonian, $H=F+\lambda V$ of Be and the correlation energy in (\%).

\begin{tabular}{ccccc}
\hline \hline$\lambda$ & DMVT $(P Q)$ & DMVT $(P Q G)$ & Full-CI & Hartree-Fock \\
\hline 0.10 & $-10.5324(249)$ & $-10.5322(100)$ & $-10.5322(100)$ & $-10.5321(0)$ \\
0.50 & $-12.3365(226)$ & $-12.3317(100)$ & $-12.3317(100)$ & $-12.3278(0)$ \\
1.00 & $-14.6064(200)$ & $-14.5895(100)$ & $-14.5895(100)$ & $-14.5725(0)$ \\
2.00 & $-19.2097(151)$ & $-19.1600(100)$ & $-19.1596(100)$ & $-19.0619(0)$ \\
3.00 & $-24.1197(115)$ & $-24.0491(100)$ & $-24.0469(100)$ & $-23.5513(0)$ \\
4.00 & $-29.6662(139)$ & $-29.2180(101)$ & $-29.2115(100)$ & $-28.0407(0)$ \\
5.00 & $-37.1084(212)$ & $-34.7026(100)$ & $-34.6922(100)$ & $-32.5301(0)$ \\
10.00 & $-76.5823(165)$ & $-68.0991(100)$ & $-68.0414(100)$ & $-54.9771(0)$ \\
10000.0 & $-81838.44(102)$ & $-81294.14(100)$ & $-81290.74(100)$ & $-44904.03(0)$ \\
\hline \hline
\end{tabular}


TABLE VIII. Total energy for the model Hamiltonian, $H=F+\lambda V$ of $\mathrm{H}_{2} \mathrm{O}$ and the correlation energy in (\%).

\begin{tabular}{lcccc}
\hline \hline$\lambda$ & $\operatorname{DMVT}(P Q)$ & DMVT $(P Q G)$ & Full-CI & Hartree-Fock \\
\hline 0.10 & $-41.1669(234)$ & $-41.1664(100)$ & $-41.1664(100)$ & $-41.1661(0)$ \\
0.50 & $-56.5293(233)$ & $-56.5158(102)$ & $-56.5155(100)$ & $-56.5051(0)$ \\
1.00 & $-75.7953(232)$ & $-75.7310(104)$ & $-75.7290(100)$ & $-75.6789(0)$ \\
1.50 & $-95.1938(235)$ & $-95.0064(106)$ & $-94.9978(100)$ & $-94.8526(0)$ \\
2.00 & $-115.0471(222)$ & $-114.5560(115)$ & $-114.4863(100)$ & $-114.0264(0)$ \\
3.00 & $-155.4737(137)$ & $-154.6553(101)$ & $-154.6348(100)$ & $-152.3740(0)$ \\
4.00 & $-196.0891(124)$ & $-195.0768(101)$ & $-195.0347(100)$ & $-190.7215(0)$ \\
5.00 & $-236.8429(120)$ & $-235.6115(101)$ & $-235.5285(100)$ & $-229.0690(0)$ \\
10.00 & $-441.1663(116)$ & $-438.6811(102)$ & $-438.3447(100)$ & $-420.8068(0)$ \\
10000.0 & $-417093.18(124)$ & $-411089.69(102)$ & $-410645.30(100)$ & $-383512.82(0)$ \\
\hline \hline
\end{tabular}

the other hand, the curves by $\operatorname{DMVT}(P Q)$ were always repulsive, which showed the potential importance of the $G$ condition.

We examined the size consistency of the present method. The $G$ condition is found to be related to the size consistency of the method and shown to be essential to the behavior of the potential curves of $\operatorname{DMVT}(P Q G)$, especially in the dissociation limit.

We also examined the Weinhold-Wilson inequalities for the 2-RDM of DMVT $(P Q G)$ calculations where the results were less accurate, and found that the inequalities VI and VII were violated. We think these inequalities may be new candidates for the $N$-representability condition of the DMVT. Since these are linear conditions, it would be easily included in the conditions of the DMVT relaxed with the SDP. Such study is now in progress.

\section{ACKNOWLEDGMENTS}

This study has been supported by the Special Grant for Scientific Research from the Japanese Ministry of Education, Science, Culture, and Sports. The authors give warm thanks to Mr. Mitsuhiro Fukuda, Dr. Katsuki Fujisawa, Dr. Kazuhide Nakata, and Professor Masakazu Kojima, for useful discussions and encouragements.

${ }^{1}$ K. Husimi, Proc. Phys. Math. Soc. Jpn. 22, 264 (1940).

${ }^{2}$ P.-O. Löwdin, Phys. Rev. 99, 1474 (1955).

${ }^{3}$ H. Nakatsuji, Phys. Rev. A 14, 41 (1976).

${ }^{4}$ C. Valdemoro, Phys. Rev. A 45, 4462 (1992); F. Colmenero, C. Pérez del Valle, and C. Valdemoro, ibid. 47, 971 (1993); F. Colmenero and C. Valdemoro, ibid. 47, 979 (1993).

${ }^{5}$ H. Nakatsuji and K. Yasuda, Phys. Rev. Lett. 76, 1039 (1996); K. Yasuda and H. Nakatsuji, Phys. Rev. A 56, 2648 (1997).

${ }^{6}$ D. A. Mazziotti, Phys. Rev. A 57, 4219 (1998); Chem. Phys. Lett. 289, 419 (1998)

${ }^{7}$ H. Nakatsuji, in Many-Electron Densities and Reduced Density Matrices, edited by J. Cioslowski (Kluwer Academic, New York, 2000).

${ }^{8}$ A. J. Coleman, Rev. Mod. Phys. 35, 668 (1963).

${ }^{9}$ C. Garrod, and J. K. Percus, J. Math. Phys. 5, 1756 (1964).

${ }^{10}$ L. J. Kijewski and J. K. Percus, Phys. Rev. A 2, 1659 (1970); L. J. Kijewski, ibid. 6, 1659 (1972); L. J. Kijewski, ibid. 9, 2263 (1974).

${ }^{11}$ C. Garrod, M. V. Mihailović, and M. Rosina, J. Math. Phys. 16, 868 (1975).

${ }^{12}$ C. Garrod and M. A. Fusco, Int. J. Quantum Chem. 10, 495 (1976).
${ }^{13}$ R. M. Erdahl, Rep. Math. Phys. 15, 147 (1979).

${ }^{14}$ R. M. Erdahl, and B. Jin, J. Mol. Struct.: THEOCHEM 527, 207 (2000); R. M. Erdahl and B. Jin, in Many-Electron Densities and Reduced Density Matrices, edited by J. Cioslowski (Kluwer Academic, New York, 2000).

${ }^{15}$ M. Nakata, H. Nakatsuji, M. Ehara, M. Fukuda, K. Nakata, and K. Fujisawa, J. Chem. Phys. 114, 8282 (2001).

${ }^{16}$ Y. Nesterov and A. S. Nemirovskii, Interior Point Polynomial Method in Convex Programming: Theory and Applications (SIAM, Philadelphia, 1993).

${ }^{17}$ L. Vandenberghe and S. Boyd, SIAM Rev. 38, 49 (1996).

${ }^{18}$ M. Kojima, Semidefinite Programming and Interior-Point Methods, http://www.is.titech.ac.jp/ kojima/wabun.html, 1996 (in Japanese).

${ }^{19}$ K. Fujisawa, M. Kojima, and K. Nakata, SDPA (SemiDefinite Programming Algorithm) User's Manual Version 5.00, August 1999. http://is-mj.archi.kyoto-u.ac.jp/ fujisawa/software.html

${ }^{20}$ D. A. Mazziotti and R. M. Erdahl, Phys. Rev. A 63, 042113 (2001).

${ }^{21}$ C. Valdemoro, L. M. Tel, and E. Perez-Romero, Phys. Rev. A 61, 032507 (2000).

${ }^{22}$ H. Nakatsuji, J. Chem. Phys. 113, 2949 (2000).

${ }^{23}$ H. Nakatsuji and E. R. Davidson, J. Chem. Phys. 115, 2000 (2001).

${ }^{24}$ H. Nakatsuji, J. Chem. Phys. 115, 2465 (2001).

${ }^{25}$ H. Nakatsuji J. Chem. Phys. 116, 1811 (2002).

${ }^{26}$ M. Ehara, M. Nakata, H. Kou, K. Yasuda, and H. Nakatsuji, Chem. Phys. Lett. 305, 483 (1999).

${ }^{27}$ F. Weinhold and E. B. Wilson, Jr., J. Chem. Phys. 47, 2298 (1967).

${ }^{28}$ E. R. Davidson, J. Math. Phys. 10, 725 (1969).

${ }^{29}$ W. B. McRae and E. R. Davidson, J. Math. Phys. 13, 1527 (1972).

${ }^{30}$ J.-P. Hansen and I. R. McDonald, Theory of Simple Liquids (Academic, Harcourt Brace, New York, 1990).

${ }^{31}$ T. V. Voorhis and M. Head-Gordon, J. Chem. Phys. 113, 8873 (2001).

${ }^{32}$ J. Paldus, P. Piecuch, L. Pylypow, and B. Jezirovski, Phys. Rev. A 47, 2738 (1993).

${ }^{33}$ S. Huzinaga, J. Chem. Phys. 42, 1293 (1970); T. H. Dunning, Jr., ibid. 53, 2823 (1970); T. H. Dunning, J. and P. J. Hay, In Method of Electronic Structure Theory, edited by H. F. Schaefer III (Plenum, New York, 1977), Vol. 2.

${ }^{34}$ Basis sets were obtained from the Extensible Computational Chemistry Environment Basis Set Database, Version 4/22/01, as developed and distributed by the Molecular Science Computing Facility, Environmental and Molecular Sciences Laboratory, the Pacific Northwest Laboratory, P.O. Box 999, Richland, WA 99352. http://www.emsl.pnl.gov:2080/forms/ basisform.html

${ }^{35}$ W. J. Hehre, R. F. Stewart, and J. A. Pople, J. Chem. Phys. 51, 2657 (1969).

${ }^{36}$ J. H. Callomon, E. Horita, K. Kuchitsu, W. J. Lafferty, A. G. Maki, and C. S. Pote, Landolt-Börnstein (Springer-Verlag, Berlin, 1976).

${ }^{37}$ K. P. Huber and G. Herzberg, Molecular Spectra and Molecular Structure IV, Electronic Constants of Diatomic Molecules (Van Nostrand Reinhold, New York, 1979).

${ }^{38}$ J. L. Dunhum, Phys. Rev. 41, 713, 721 (1932); H. M. Hulburt and J. O. Hirschfelder, J. Chem. Phys. 9, 61 (1941). 